\title{
Microscopic investigation of laser-induced structural changes in single-wall carbon nanotubes
}

\author{
Harald O. Jeschke, ${ }^{1, *}$ Aldo H. Romero, ${ }^{2}$ Martin E. Garcia, ${ }^{3}$ and Angel Rubio ${ }^{4}$ \\ ${ }^{1}$ Institut für Theoretische Physik, Universität Frankfurt, Max-von-Laue-Strasse 1, 60438 Frankfurt, Germany \\ ${ }^{2}$ CINVESTAV Querétaro, Libramiento Norponiente No. 2000, 76230 Querétaro, Mexico \\ ${ }^{3}$ Theoretische Physik, FB 18, Universität Kassel and Center for Interdisciplinary Nanostructure Science and Technology (CINSaT), \\ Heinrich-Plett-Strasse 40, 34132 Kassel, Germany \\ ${ }^{4}$ Física de Materiales, Facultad de Ciencias Químicas, Universidad del País Vasco, Centro Mixto UPV-CSIC, and European Theoretical \\ Spectroscopy Facility (ETSF), E-20018 San Sebastián/Donostia, Spain \\ (Received 5 August 2006; revised manuscript received 30 November 2006; published 12 March 2007)
}

\begin{abstract}
Extensive excited-state molecular dynamics simulations of femtosecond laser-induced structural transformation in single-walled carbon nanotubes (SWNTs) are presented. We considered in the simulation two limiting cases; one where only a short portion of the tube is irradiated and the other where the whole tube is affected by the laser pulse. We have analyzed the role of chirality (zigzag versus armchair and some chiral tube cases) in the damage threshold as a function of tube diameter. Nontrivial dependence of the damage threshold as a function of diameter has been found. We find that for equal laser parameters, zigzag SWNTs are on average equally stable with respect to laser excitation as armchair SWNTs, but their stabilities show different dependencies on diameter. Due to the higher stiffness of the $(n, n)$ tubes in the direction perpendicular to its axis as compared to the $(n, 0)$ tubes, we find the formation of standing waves in the nanotube wall for zigzag and not for armchair tubes. We also studied the role of the laser pulse duration and show that in general longer laser pulses increase the damage threshold. This result is rationalized in terms of electron-ion relaxation times. Implications of laser-induced structural transformations are analyzed.
\end{abstract}

DOI: 10.1103/PhysRevB.75.125412 PACS number(s): 78.70. $-\mathrm{g}$, 52.38.Mf, 61.46.Fg, 71.15.Pd

\section{INTRODUCTION}

Carbon nanotubes are one of the most interesting materials at the nanoscale range. They have received a lot of attention due to their excellent thermal, electronic, and optical properties which are promising nanodevice applications in the the near future. ${ }^{1-4}$ As electron sources, they have a large electron conductivity and field electron emission. ${ }^{4}$ These amazing properties are usually found in experiments performed on individual nanotubes, resulting in a difficulty in scaling those measurements when they are applied to a bunch of nanotubes. This means that the electronic properties of these systems depend crucially on the nanotube quality. This dependence is mainly due to the presence of absorbed molecules ${ }^{5}$ and vacancies or defects ${ }^{6}$ as they deteriorate their electronic and thermal properties. Therefore it is important to find experimental methods which help to improve the nanotube quality and improve their electron field emission properties. In this respect, different approaches have been taken, starting from applying external fields ${ }^{7}$ during the nanotube growth up to fine tuning the growth conditions with physical parameters such as temperature. ${ }^{8}$

In recent years, femtosecond and picosecond laser pulses have been shown to be a very useful tool to change, manipulate, calibrate or create new structures at small length scales ranging from millimeters to nanometers depending on the laser wavelength. ${ }^{9-13}$ This is mainly due to the fact that laser pulses are able to deposit a large amount of energy in a small spatial region while the temporal scale between the energy deposition and the ion vibrations are comparable. The energy deposited is absorbed by the electrons and after some electron-electron relaxation which happens on a very short time scale, the energy is transferred to the ions in its own time scale. In the femtosecond regime, it is possible to observe, among others, atoms or molecules in the course of transformations, chemical reactions, biological processes, and catalytic processes.

Several experimental studies on laser irradiation of carbon nanotubes have been published. Recently, a comparison between thermal heating and low laser irradiation intensity has been reported for multiwall carbon nanotubes. ${ }^{8}$ In thermal treatment, the authors have found an improvement of the electronic emission as a function of temperature (of the order of $700{ }^{\circ} \mathrm{C}$ ) but they also found a large degradation on the nanotube quality. Instead, in the case of a laser excitation, the electronic emission is increased almost as efficiently as in the thermal case but the nanotube quality is also improved, this being mainly due to the fact that laser irradiation does not cause a permanent damage to the nanotubes. Their observations show that laser fields have more beneficial influence on the nanotube quality and its transport properties after laser application than thermal treatments. Smalley and co-workers have performed fluorescence measurements with femtosecond laser pulses and they have found a very fast electronic relaxation (at most of $100 \mathrm{fs})^{14}$ and fluorescence emission (about $10 \mathrm{ps).}{ }^{15} \mathrm{Ma}$ and co-workers ${ }^{16}$ have studied the morphology changes on carbon nanotubes under laser irradiation. In this particular case, the laser was of low intensity (of the order of $3 \times 10^{4} \mathrm{~W} / \mathrm{cm}^{2}$ ). They observed very important changes on the nanotube structure characterized by studying TEM images before and after the laser application. More recently, Dresselhaus and co-workers ${ }^{17}$ have studied experimentally, possible morphological changes on carbon nanotubes. After laser irradiation and depending on the laser parameters an improvement of structural ordering and perhaps removal of chemisorbed gases has been reported. This study was based on Raman spectroscopy measurements on nano- 
tube samples after laser deposition. At low intensity irradiation, the nanotubes can be sintered together, whereas at increased itensity, the nanotubes get damaged and an important reconstruction has been observed. A very important observation was a dependence of the threshold damage intensity as function of the tube diameter. Even though they did not explain this dependence microscopically, they reported that smaller diameter tubes are burnt off at lower laser intensities. Resonant Raman spectroscopy is an excellent tool for the investigation of laser-induced structural modifications of carbon nanotube ensembles. ${ }^{18}$ Structural changes in singlewalled carbon nanotubes (SWNTs) have also been investigated after heat treatment. ${ }^{19}$ On the theoretical side, besides the characterization of the optical absorption of carbon nanotubes,${ }^{20}$ photodesorption has been proposed as a noninvasive tool to clean carbon nanotubes, ${ }^{21}$ vibrational excited state dynamics has been shown to be a very reliable tool to identify topological defects in carbon tubes, ${ }^{12,22}$ and selective cap opening of carbon nanotubes induced by femtosecond lasers was predicted. ${ }^{23}$

Motivated by these experiments we have performed a set of calculations of femtosecond laser pulses applied on single-wall carbon nanotubes. We present results of the threshold energy required to damage the nanotube as function of tube chirality, diameter, and laser pulse duration. We find that the damage thresholds are complex nonmonotonous functions of the diameters of nanotubes. For one fixed diameter, we find a threshold that is nearly independent of the nanotube chirality.

This paper is organized as follows. First we briefly describe the theoretical approach we use to handle the excited state electron-ion dynamics in the presence of a laser pulse, then we discuss the results for the damage thresholds for zigzag and armchair nanotubes having different diameters. Finally, we conclude by summarizing our results.

\section{THEORY}

We simulate laser-induced ultrafast structural changes in carbon nanotubes using a method based on nonadiabatic molecular dynamics simulations. We take into account, on a microscopic level, the laser excitation and thermalization processes sustained by the electrons and their influence on the lattice dynamics. The method has been described in detail in Ref. 24 and applied to carbon solids and nanostructures. ${ }^{12,23,25-27}$ The problem of nonadiabatic molecular dynamics has also been treated with methods of surface hopping ${ }^{28-30}$ and the quantum-classical mean field (Ehrenfest) method. ${ }^{31-33}$ In the following we summarize the main points of our approach. The movement of the atoms is described by integrating the equations of motion, which are obtained from the Lagrangian ${ }^{34,35}$

$$
L=\sum_{i=1}^{N} \frac{1}{2} m_{i} \dot{\boldsymbol{s}}_{i}^{t} h^{t} h \dot{\boldsymbol{s}}_{i}-U\left(\left\{r_{i j}\right\}\right)+\frac{1}{2} W \operatorname{Tr}\left(\dot{h}^{t} \dot{h}\right)-p_{\mathrm{ex}} \operatorname{det}(h) .
$$

Here, the first two terms in the Lagrangian yield the Newton equations of motion for the atoms while the last two terms lead to equations of motion for the additional degrees of freedom, which in this case correspond to the lattice parameters of the MD supercell. In particular, the third term represents the contribution to the total kinetic energy coming from the alteration of the primitive vectors. This term is only nonzero if the MD supercell changes its shape and/or volume during the simulation. The external pressure $p_{\text {ex }}$ acts on the MD supercell which can change its shape and volume according to its equations of motion. $U\left(\left\{r_{i j}\right\}\right)$ is the potential energy surface (PES) on which atoms are moving. $r_{i j}=\mid \vec{r}_{i}$ $-\vec{r}_{j} \mid$ refers to the interatomic distance between atoms $i$ and $j$. The absolute coordinates of the atoms $\boldsymbol{r}_{i}$ are connected via $\boldsymbol{r}_{i}=h \boldsymbol{s}_{i}$ to the relative coordinates $\boldsymbol{s}_{i}$ by the matrix $h$ that contains the vectors spanning the MD supercell which contains the atoms being considered.

The PES is calculated as the free energy of the valence electrons

$$
U\left[\left\{r_{i j}\right\},\left\{n_{l}\right\}\right]=V_{e}\left[\left\{r_{i j}\right\},\left\{n_{l}\right\}\right]-T_{e} S_{e}\left[\left\{n_{l}\right\}\right],
$$

and is a function of the interatomic distances $\left\{r_{i j}\right\}$ and the occupations of the electronic levels $n_{l}$. In Eq. (2), the quantity $V_{e}$ represents the energy of the system, $V_{e}=V_{\mathrm{TB}}+V_{\text {rep }}$, where the first term is the attractive (bonding) energy due to the valence electrons and the second term stands for the repulsive interaction between the atomic cores. $V_{\mathrm{TB}}$ is obtained from the diagonalization of an orthogonal tight binding (TB) Hamiltonian of the form

$$
H=\sum_{i \alpha} \epsilon_{i \alpha} n_{i \alpha}+\sum_{\substack{i j \alpha \beta \\ j \neq i}} V_{i j}^{\alpha \beta}\left(r_{i j}\right) c_{i \alpha}^{+} c_{j \beta},
$$

where $\epsilon_{i \alpha}$ is the on-site energy of atom $i$ and orbital $\alpha . c_{i \alpha}^{+}$ and $c_{j \alpha}$ are the creation and annihilation operators, and $V_{i j}^{\alpha \beta}\left(r_{i j}\right)$ the hopping integrals. For the description of carbon, the $2 s, 2 p_{x}, 2 p_{y}$, and $2 p_{z}$ orbitals are taken into account. For the interatomic distance dependence of these hopping integrals and for $V_{\text {rep }}$ we employ the forms proposed by Xu et $a l^{36}$

The second part of the right hand side of Eq. (2) describes the entropy term of the electron free energy. $S_{e}$ $=-2 k_{\mathrm{B}} \Sigma_{l}\left[n_{l} \ln \left(n_{l}\right)+\left(1-n_{l}\right) \ln \left(1-n_{l}\right)\right]$ is the electronic entropy and $T_{e}$ the electronic temperature.

At low temperatures and in the absence of an external field the entropy term in the PES is negligible and the atoms move on a potential determined by the electronic ground state and the repulsive core-core interaction. However, when a time dependent laser field of shape $E(t)$ and frequency $\omega$ excites the system, it excites electron-hole pairs and therefore produces changes in the electronic occupations $n_{l}$. Thus, due to the presence of an ultrashort laser pulse and the subsequent thermalization process the occupations $n_{l}(t)$ become time dependent quantities. This leads to a time dependence of the electronic temperature $T_{e}(t)$ and the electronic entropy $S_{e}(t)$. Already for moderate intensities of the laser pulse the changes in the occupations are such, that the entropy term of the PES starts to play a very important role. 
To describe the light absorption and thermalization processes which cause the changes in the $n_{l}$ 's we solve a set of coupled equations of motion for the occupations, which are derived from the Liouville-von Neumann equation for the reduced density matrix. Thus, the time derivatives of the $n_{l}$ 's satisfy ${ }^{24}$

$$
\begin{aligned}
\frac{d n\left(\epsilon_{m}, t\right)}{d t}= & \int_{-\infty}^{\infty} d \omega g(\omega, t-\Delta t)\left\{\left[n\left(\epsilon_{m}-\hbar \omega, t-\Delta t\right)+n\left(\epsilon_{m}\right.\right.\right. \\
& \left.\left.+\hbar \omega, t-\Delta t)-2 n\left(\epsilon_{m}, t-\Delta t\right)\right]\right\}-\frac{n\left(\epsilon_{m}, t\right)-n^{0}\left(\epsilon_{m}\right)}{\tau_{1}} .
\end{aligned}
$$

Here, the first term describes the excitation process, with the spectral function of the laser pulse $g$. The second term of Eq. (4) describes the electron-electron collisions that lead to an equilibration of the electronic system with a rate equation of the Boltzmann type for the occupation $n\left(\epsilon_{m}, t\right)$. Hence, with a time constant $\tau_{1}$, the occupation $n\left(\epsilon_{m}, t\right)$ approaches a FermiDirac distribution $n^{0}\left(\epsilon_{m}\right)$. In other words, the laser pulse changes the initial ground state electronic distribution $n_{l}\left(\mu_{0}, T_{0}\right)$ into a time dependent nonequilibrium distribution $n_{l}(t)$, which then converges in a time scale of $\tau_{1}$, due to electron-electron collisions, to a Fermi-like function $n_{l}\left(\mu, T_{e}\right) . \mu(t)$ is the chemical potential. We model the action of the laser pulse by assuming a spatially uniform excitation of the SWNT section in the MD supercell. This might seem at odds with the fact that typical experimental laser profiles have a Gaussian intensity distribution in space. But it becomes plausible if we consider the length scales involved: While a typical laser spot is several micrometers in diameter, the SWNT sections we simulate measure a few nanometers. The change in intensity of a Gaussian pulse with width $\sigma$ over a tube section of length $l$ is proportional to $(l / \sigma)^{2}$. This gives a change of $10^{-6}$ if $l \sim 1 \mathrm{~nm}$ and $\sigma \sim 1 \mu \mathrm{m}$. Thus the electric field inside the MD supercell is approximately uniform, and it is justifiable to use periodic boundary conditions as even the SWNT sections neighboring the primary MD supercell will experience similar electric field strengths. Furthermore, the wavelength of the laser light greatly exceeds the dimensions of the simulated nanotube section. In order to simulate the effects of the laser in different regions of the Gaussian intensity profile, we have to employ several of our simulations with small supercells and uniform excitation in order to sample the different intensity regions of the laser pulse profile.

Molecular dynamics simulations require determination of the forces $\mathbf{F}_{j}$ acting on the $j$ th ion. This is done using the Hellman-Feynman theorem.

In our discussion of the interaction between the nanotube and the external femtosecond laser pulse we distinguish two different kinds of boundary conditions along the tube axis, which correspond to two different physical situations: (i) variable size supercell and (ii) fixed size supercell. The condition (i) is used when the CNT is completely contained in the spot irradiated by the laser pulse. In this case the diameter of the laser spot is larger than the length of the nanotube [see Fig. 1(a)], and the electronic excitation produced by the
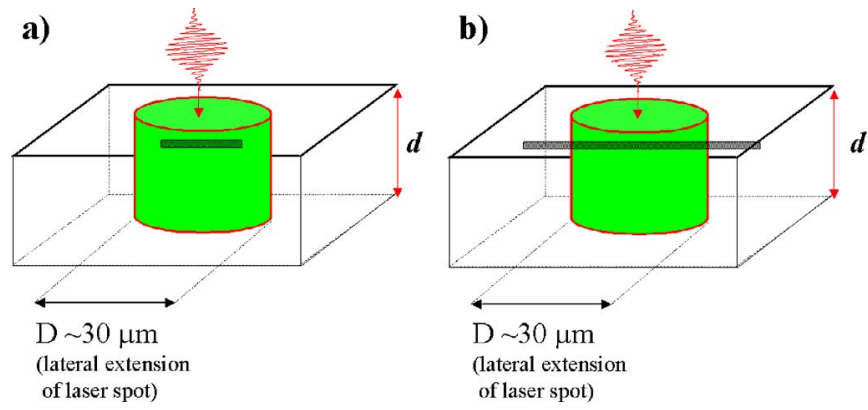

FIG. 1. (Color online) Schematic illustration of the two different types of boundary conditions used in the MD simulations on the nanotubes.

laser pulse can lead to an ultrafast expansion of the entire tube. It is important to point out that in this situation the expansion velocity of the tube is not limited by the sound velocity, since the whole nanotube is excited and the ground state potential energy surface (on which the sound velocity is defined) can be dramatically changed. ${ }^{35}$ The tube can expand in the longitudinal direction after the action of the laser pulse. The condition (ii) is used to simulate a local excitation of the nanotube by the laser, which will occur when the diameter of the laser spot is smaller than the length of the nanotube [see Fig. 1(b)]. In this case, the irradiated part of the nanotube cannot undergo ultrafast longitudinal expansion, since the expansion velocity is limited by the sound velocity of the unexcited part. We assume there is no longitudinal expansion and we use fixed periodic boundary conditions (the unit cell is not permitted to change, putting a restriction on the longitudinal expansion of the CNT). In the directions perpendicular to its axis, the CNT is surrounded by ample amounts of vacuum, thus permitting any radial expansion the potential energy surface demands. With the fixed size supercell, we are simulating the limiting case of an SWNT that is completely prevented from length fluctuations. While this extreme may not be exactly realizable in an experimental situation, we believe that consideration of this limit is valuable to highlight differences in SWNT fragmentation behavior induced by the experimental setup.

In the following we define the damage threshold as the laser intensity necessary to produce an irreversible change in the structure of the nanotube. We should clarify at this point that we do not have a linear relation between the maximum amplitude of the laser field $E(t)$, which represents the external electromagnetic field, and the energy $E_{0}$ absorbed by the electrons. ${ }^{37}$ Nevertheless, the convolution between the laser's intensity distribution and the population number determines the absorbed energy. The laser field is given by a localized oscillatory wave with a given intensity and a time lag, as mimicked in Fig. 1. At the end, the absorbed energy depends on how excited states are populated by the electrons.

\section{RESULTS}

To facilitate the presentation and discussion of the extensive molecular dynamics simulations performed in this study, 

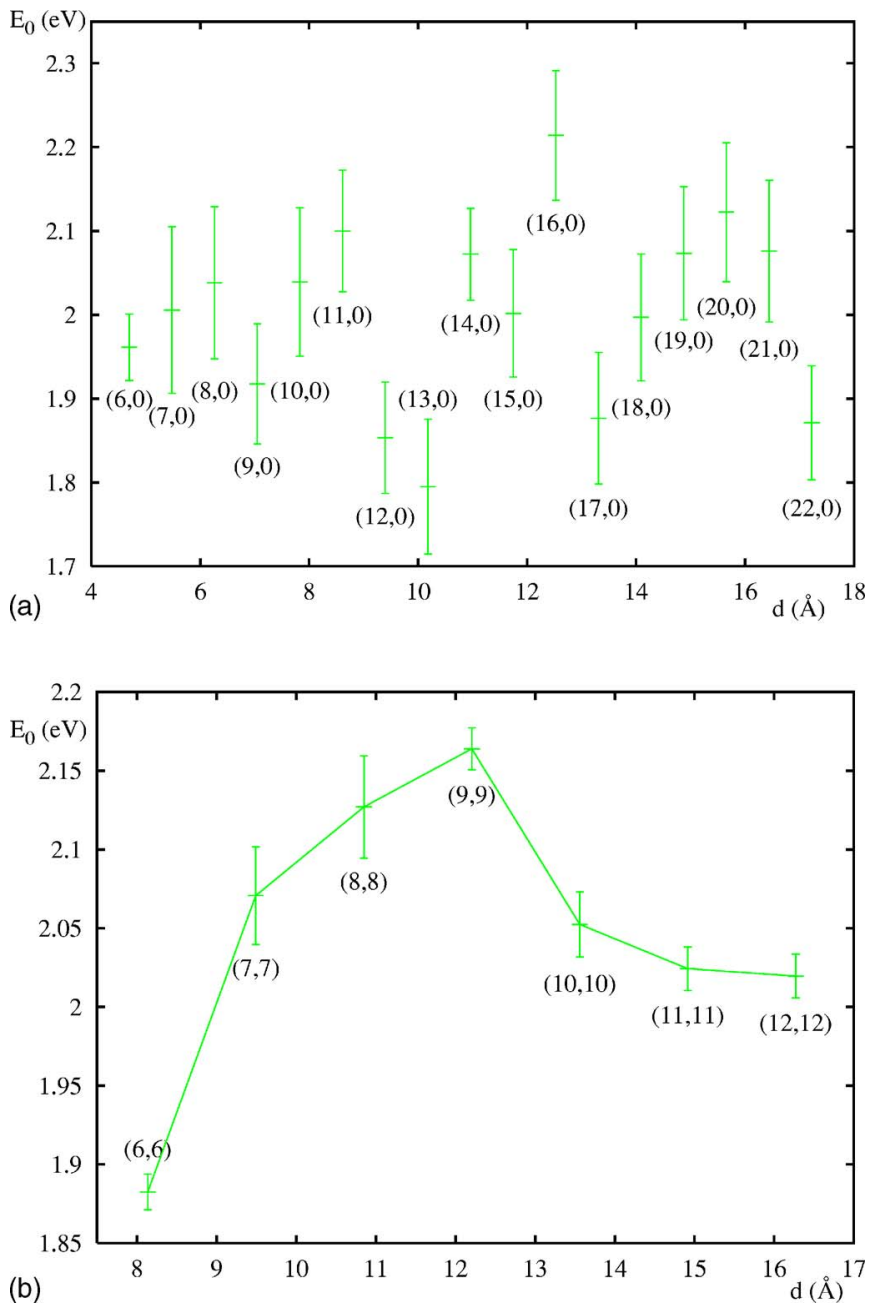

FIG. 2. (Color online) Damage thresholds of zigzag (a) and armchair (b) SWNTs as a function of tube diameter. The damage threshold is defined as the minimum energy deposited by the laser in order to produce an irreversible structural transformation of the tube. Both panels correspond to fixed periodic boundary conditions. This corresponds to long nanotubes that are only partially excited by the laser pulse and thus cannot expand in the longitudinal direction. Note that for zigzag tubes, the duration of the laser pulse was $\tau=20 \mathrm{fs}$, whereas it was $\tau=50$ fs for armchair tubes.

we divide the discussion into two sections depending on the type of boundary condition used to address the laser-induced structural modifications of the tube (see above). In the two cases we analyze the results for armchair and zigzag carbon nanotubes of different diameters in terms of the laser pulse applied, in particular we focus on the how the structure changes as a consequence of the energy absorbed by the tube during the laser excitation and how this depends on the laser pulse duration (from pulses of $\tau=20$ to $100 \mathrm{fs}$ ). In all the results the key quantity we looked at is the damage threshold (as introduced in the previous section), as a function of diameter. In this context, Figs. 2 and 4 summarize the results of the present work for the damage thresholds of zigzag and armchair CNTs for fixed and variable boundary conditions. Now we are going to comment separately on the results obtained for each of the two types of boundary conditions that we have used.

\section{A. Fixed boundary conditions}

As discussed above this geometry would correspond to long tubes supported on a substrate and possibly held in place by bulk material that prevents the tube from expanding on time scales shorter than those determined by the sound velocity. In this configuration we take the spot of the laser pulse smaller than the tube length, which therefore acts only at the central region of the tube. Nevertheless, we assume the laser intensity to be homogeneous in the short tube section in our simulation supercell. For the different tube diameters and chiralities we have considered, different supercell sizes were used according to computational necessity. In particular, the shortest nanotube we have considered is $12.3 \AA$ in length [with chirality $(6,6)]$ and the longest one is $33.8 \AA$ in length [with chirality $(8,4)]$. The number of atoms ranges from $N$ $=120$ to $N=352$.

In Fig. 2 we summarize the results for the damage thresholds of zigzag and armchair CNTs with fixed boundary conditions. As a general observation, the damage threshold does not have monotonous dependence on tube diameter. For the studied diameters, the average damage threshold is $E_{0}$ $=2.00 \pm 0.09 \mathrm{eV} /$ atom for zigzag tubes with fixed boundary conditions at $\tau=20 \mathrm{fs}$ pulse duration, and $E_{0}$ $=2.05 \pm 0.07 \mathrm{eV} /$ atom for armchair nanotubes at $\tau=50 \mathrm{fs}$ pulse duration. Furthermore, the maximum threshold is obtained for tube diameters of about $d=12 \AA$ in both cases. This structure is more clear for armchair tubes than zigzag tubes that exhibit a more oscillatory dependence with tube diameter. Towards the lower diameters we find a decrease of stability that would agree with the observation that nanotubes of higher curvature tend to be less stable. ${ }^{38}$

We illustrate how the structural damage is induced in the tube by showing in Fig. 3 the computed trajectory for the $(22,0)$ SWNT for laser deposited energy of $E_{0}$ $=2.45 \mathrm{eV} /$ atom (pulse duration $\tau=20 \mathrm{fs}$ ) that is $30 \%$ above the damage threshold for this tube in Fig. 2. Upon laser excitation, we observe that irreversible damage occurs within the first picosecond. The process of fragmentation starts with the emission of some carbon monomers few hundred femtoseconds after the laser pulse, which leads to a large tear in the CNT wall and to the formation of some linear carbon chains. This observation is in qualitative agreement with a previous work, where a concerted "patching-and-tearing" mechanism was proposed $^{39}$ to explain the transition from single-walled nanotube ropes to multiwall nanotubes upon heat treatment. We could envision here that a similar process could be obtained by a short-laser pulse. The laser could trigger such a structural transformation of single-walled ropes to multiwall nanostructures, or other modifications (by shaping the laser pulse and its duration).

\section{B. Variable cell simulations}

This setup has been chosen with the idea of treating tubes that are irradiated by a laser pulse in all their length. In this case we allow the relaxation of the boundaries since ultrafast expansion is possible. This opens the possibility of energy transfer into longitudinal oscillations, which propagate along the carbon nanotube. This could be an important part in a 

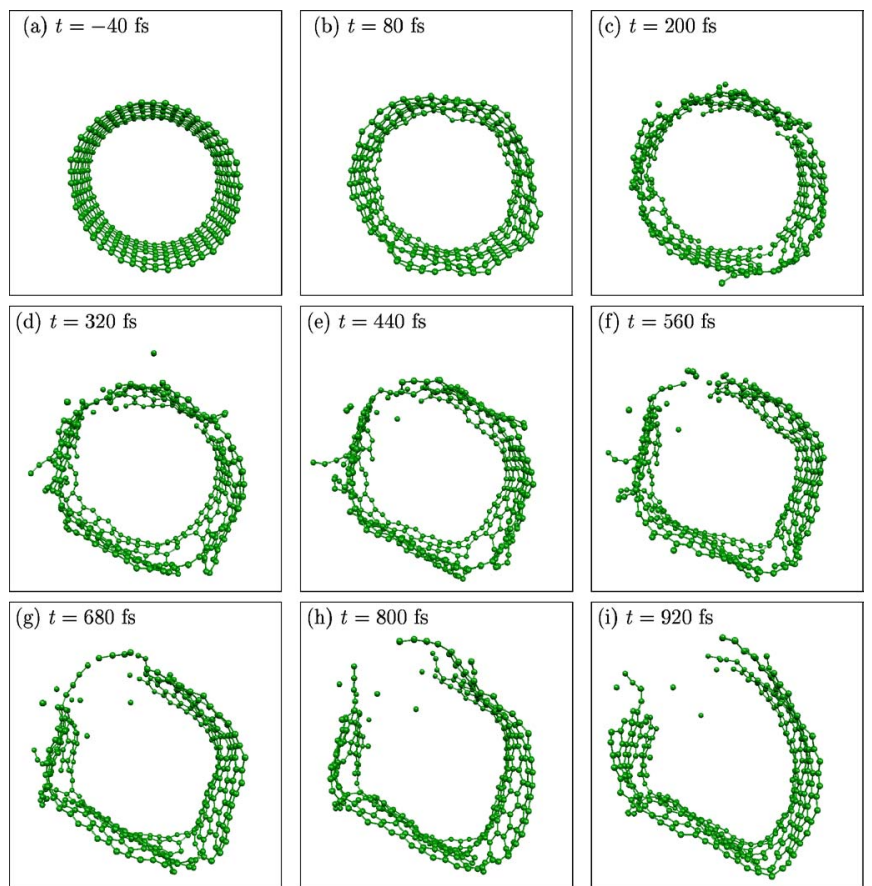

FIG. 3. (Color online) Snapshots of the time evolution of a $(22,0)$ SWNT after laser excitation $30 \%$ above the damage threshold. The duration of the laser pulse was $\tau=20 \mathrm{fs}$, and the deposited energy $E_{0}=2.45 \mathrm{eV} /$ atom. Longitudinal expansion of the tube upon laser excitation was not permitted by fixing the boundary conditions.

mechanism to distribute the absorbed energy together with the standard mechanical (plastic or brittle) deformation discussed above in the case of fixed boundary conditions.

Figure 4 summarizes the results for the damage thresholds of zigzag and armchair CNTs with variable boundary conditions. In this case there is no clear maximum for the damage threshold and the two types of tubes behave similarly, with diameter fluctuations in the zigzag smaller than in the armchair case (as in the case of fixed boundary conditions). Here, the average threshold for damage is higher since for variable boundary conditions additional relaxation channels appear, namely, a longitudinal wave propagation. The average value $E_{0}=2.09 \pm 0.10 \mathrm{eV} /$ atom for zigzag tubes with variable boundary conditions at $\tau=20 \mathrm{fs}$ pulse duration for the tube diameter range shown in Fig. 4(a) is above the corresponding value of $E_{0}=2.00 \pm 0.09 \mathrm{eV} /$ atom for the same nanotubes with fixed boundary conditions [see Fig. 2(a)]. The average damage threshold for the armchair nanotubes of Fig. 4(b) is $E_{0}=2.21 \pm 0.04 \mathrm{eV} /$ atom, also higher than the average value $E_{0}=2.05 \pm 0.07 \mathrm{eV} /$ atom corresponding to Fig. 2(b).

To get more insight into the dynamics of the damage and the role of the laser pulse parameters, we explore the influence of the pulse duration. In Fig. 5 we show the damage thresholds of armchair CNTs with variable periodic boundary conditions for pulse durations of $\tau=20,50$, and $100 \mathrm{fs}$. The overall picture is that the longer the laser pulse the larger the damage threshold. We find an exception to this trend for the $(7,7)$ CNT. The average thresholds for the diameter ranges shown in Fig. 5 are $E_{0}=2.09 \pm 0.07 \mathrm{eV} /$ atom for $\tau$
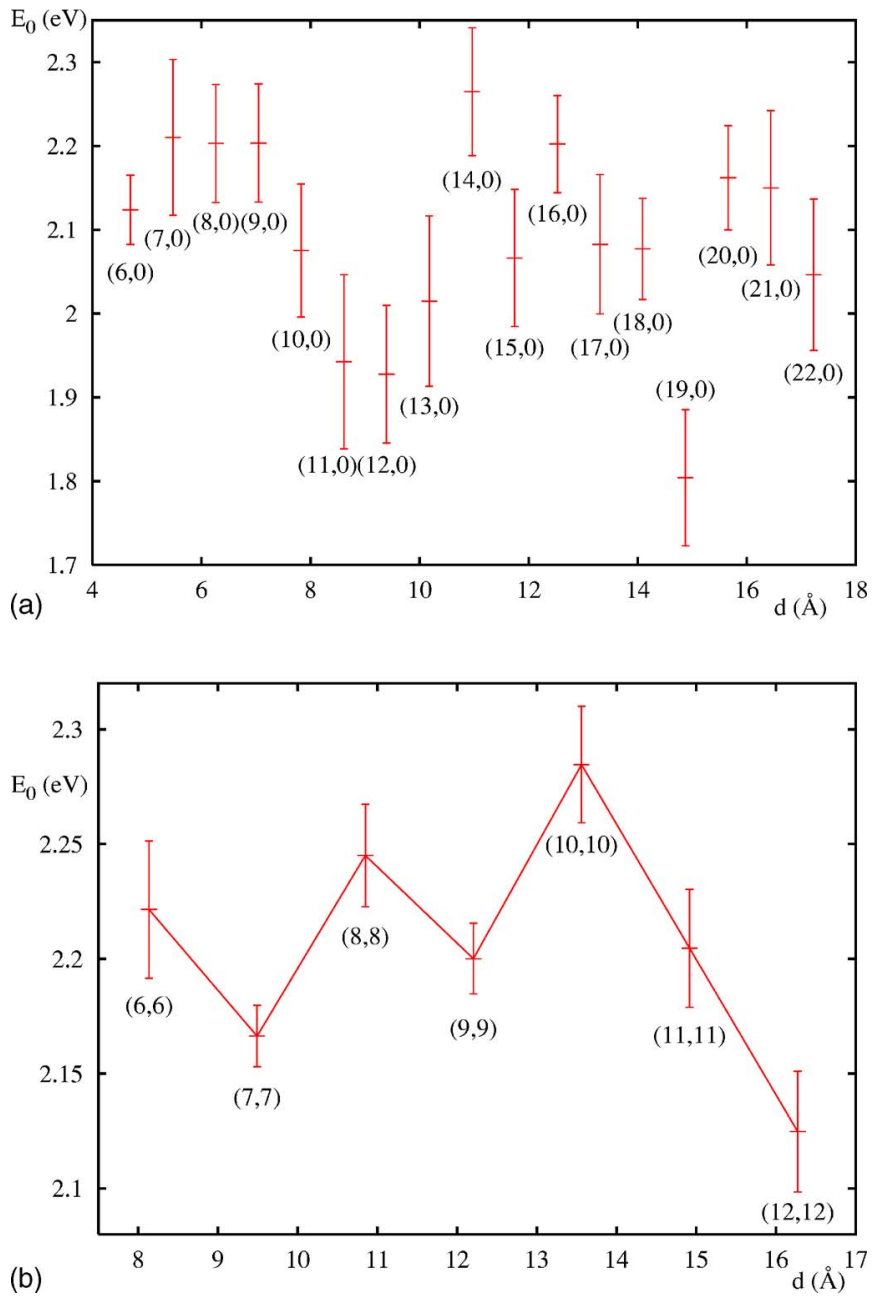

FIG. 4. (Color online) Damage thresholds of zigzag (a) and armchair (b) SWNTs as a function of tube diameter. Both panels correspond to variable periodic boundary conditions. This corresponds to short nanotubes that fit entirely into the laser spot and can thus expand freely upon laser excitation. Note that for zigzag tubes, the duration of the laser pulse was $\tau=20 \mathrm{fs}$ whereas it was $\tau$ $=50 \mathrm{fs}$ for armchair tubes.

$=20 \mathrm{fs}, \quad E_{0}=2.21 \pm 0.04 \mathrm{eV} /$ atom for $\tau=50 \mathrm{fs}$, and $E_{0}$ $=2.37 \pm 0.11 \mathrm{eV} /$ atom for $\tau=100 \mathrm{fs}$ pulse duration. For longer pulse durations, the CNT is able to accommodate a larger amount of laser energy without sustaining damage. This result can be rationalized in terms of electron-ion relaxation: For short laser pulses the intensity is very high and does not give the system time for structural relaxation to accommodate the additional electronic energy provided by the laser. As the pulse duration becomes comparable to the time scale for structural relaxation, the nanotube can redistribute part of the energy among different degrees of freedom while it is flowing into the system. In the limit of infinite pulse duration one expects the absorbed energy to be distributed among all degrees of freedom according to the equipartition theorem.

In addition, by comparing the effect of the same laser pulse on zigzag and armchairs tubes [compare Figs. 4(a) and 5 for $\tau=20 \mathrm{fs}$ ] we observe that on average, zigzag, and armchair tubes have comparable damage thresholds. It is inter- 


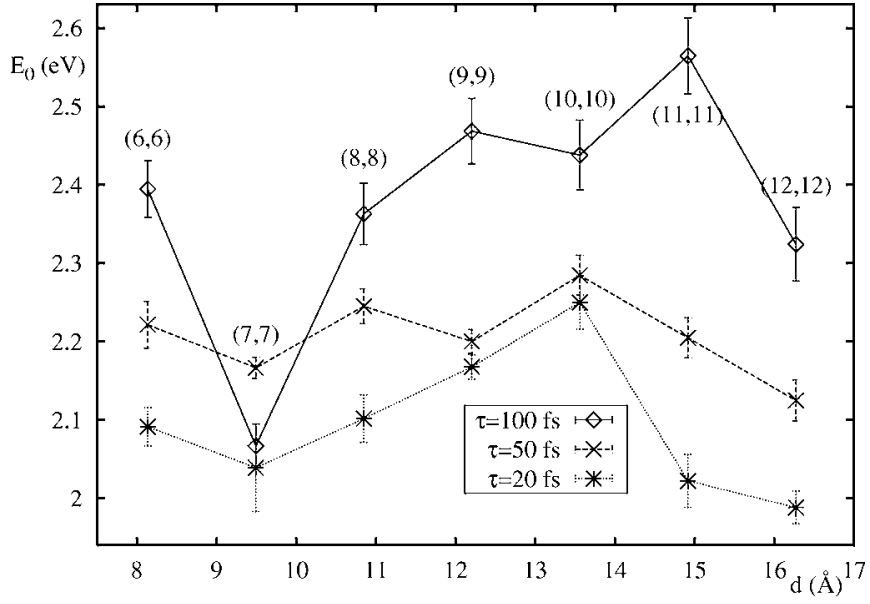

FIG. 5. Damage thresholds of $(n, n)$ SWNTs as a function of tube diameter for variable tube length (see text). The duration of the laser pulses was varied from $\tau=20 \mathrm{fs}$ (star symbols, dotted error bars) over $\tau=50 \mathrm{fs}$ (cross symbols, dashed error bars) to $\tau=100 \mathrm{fs}$ (diamond symbols, solid error bars).

esting to point out that though the overall response of armchair and zigzag nanotubes is different, we found particular diameters for which the damage threshold was almost independent of the chirality. In Fig. 6 we show results for the damage thresholds of tubes with $d=8 \AA$ diameter but chiralities between $0^{\circ}$ and $45^{\circ}$.

Nanotubes are experimentally known to possess structural defects. While the main focus of our investigation was the structural response of perfect SWNTs, we have for a few sample tube geometries determined how the thresholds would change if a single vacancy were present. We find for $(10,0),(15,0)$, and $(20,0)$ with a high linear vacancy concentration of $n_{\mathrm{def}}=0.06 \AA^{-1}$ ablation thresholds of $E_{0}$ $=1.84 \pm 0.04 \mathrm{eV} /$ atom, $1.79 \pm 0.05$, and $1.85 \pm 0.05 \mathrm{eV} /$ atom, respectively. This should be compared to the corresponding

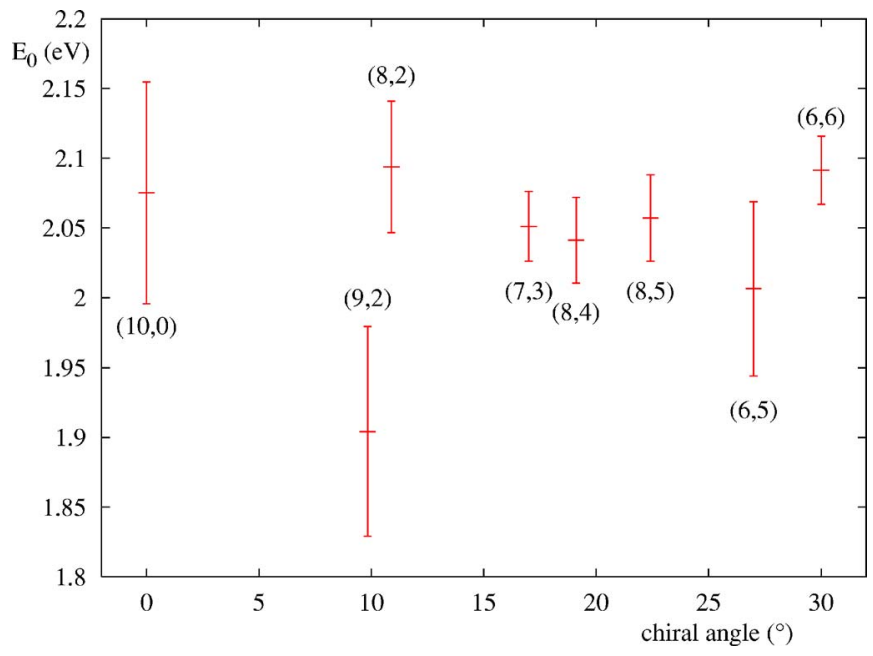

FIG. 6. (Color online) Damage thresholds for chiral tubes as a function of chiral angle for a diameter $d \approx 8 \AA$. Note that the variation of thresholds is smaller than in Figs. 2 and 4 . Variable boundary conditions allowed the tube length to fluctuate. The laser pulse duration was $\tau=20 \mathrm{fs}$.
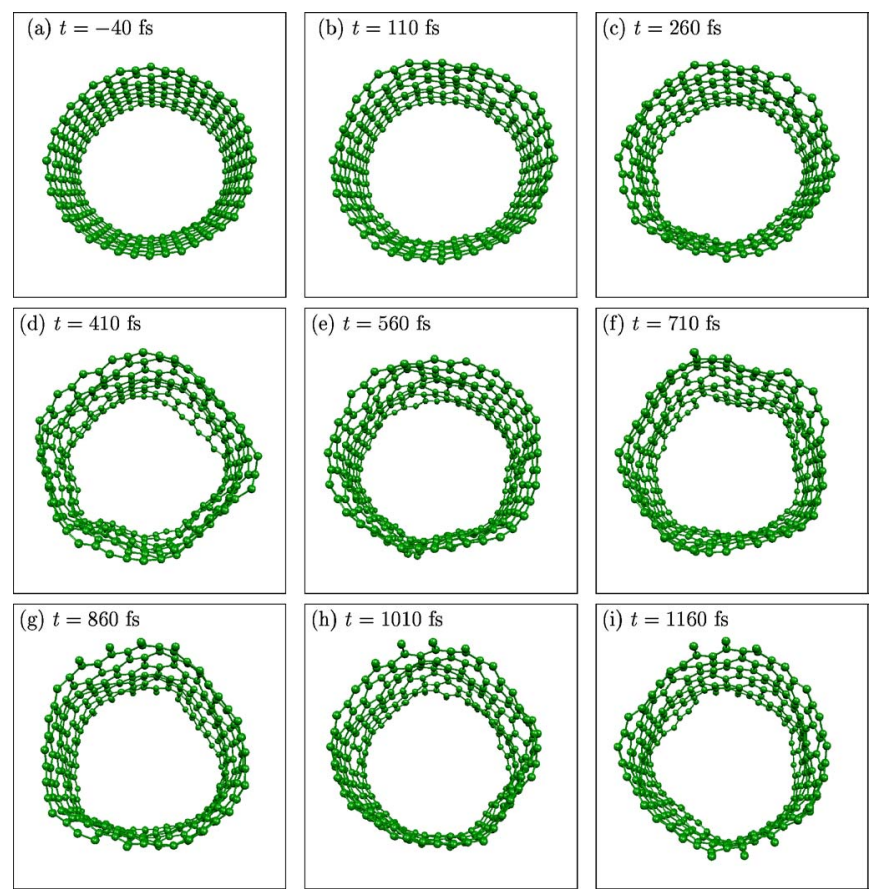

FIG. 7. (Color online) Snapshots of the time evolution of a $(20,0)$ SWNT after laser excitation slightly below the damage threshold. The duration of the laser pulse was $\tau=20 \mathrm{fs}$, the deposited energy $E_{0}=2.1 \mathrm{eV} /$ atom. Longitudinal expansion of the tube upon laser excitation was permitted.

values for the perfect tubes (see Fig. 4) $E_{0}=2.07 \pm 0.08$, $2.07 \pm 0.08$, and $2.16 \pm 0.07 \mathrm{eV} /$ atom, respectively. Thus we find that the damage threshold is lowered by approximately $0.2 \mathrm{eV} /$ atom or $10 \%$ due to the presence of a single vacancy. In previous investigations of SWNTs with pentagonheptagon defects ${ }^{12,40}$ we have found a similar decrease of the damage threshold of roughly $10 \%$ in the presence of defects.

We complete the study by presenting an analysis of particular trajectories for different absorbed energies, focusing on structural changes close to and below the damage threshold. In Fig. 7, the $(20,0)$ SWNT with variable boundary conditions remains intact after absorption of $E_{0}=2.1 \mathrm{eV} /$ atom (pulse duration $\tau=20 \mathrm{fs}$ ). Upon laser excitation, we observe a strong radial expansion and, several hundred femtoseconds after the pulse some breathing vibrational excitation of the SWNT wall. The most remarkable feature of the vibrational excitation is a standing wave that forms on the CNT wall and which will be analyzed in more detail below.

Slightly above the damage threshold, i.e., for $E_{0}$ $=2.35 \mathrm{eV} /$ atom, the damage occurring within the first picosecond after the action of the laser pulse of $\tau=20$ fs duration consists only in the emission of carbon monomers (see Fig. 8). If the intensity is increased further we see the same behavior as discussed in the previous section for fixed boundary conditions (see Fig. 3).

\section{DISCUSSION}

After having presented the results for both boundary conditions, we now analyze them in more detail. The first im- 


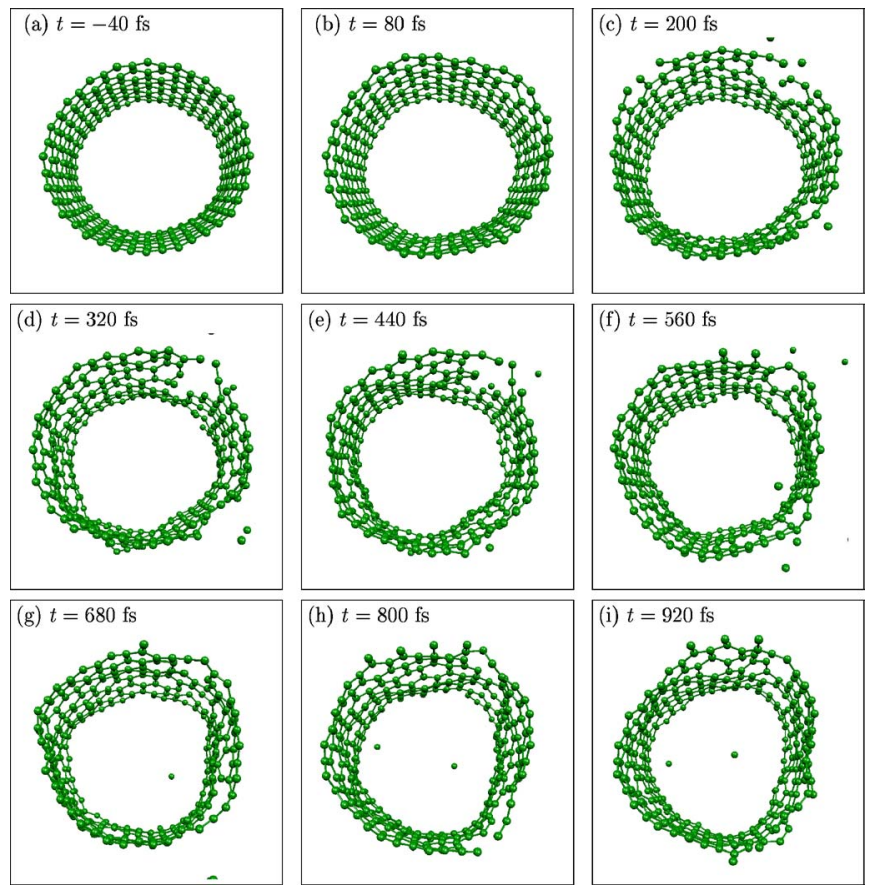

FIG. 8. (Color online) Snapshots of the time evolution of a $(20,0)$ SWNT after laser excitation slightly above the damage threshold. The duration of the laser pulse was $\tau=20 \mathrm{fs}$, the deposited energy $E_{0}=2.35 \mathrm{eV} /$ atom. Longitudinal expansion of the tube upon laser excitation was permitted. Several atoms are emitted and leave the displayed area.

portant point from the previous results is that damage thresholds do not show a simple monotonous behavior as a function of tube diameter. It is important to keep in mind that the damage thresholds calculated in our study correspond to damage in the presence of a massive electron-hole excitation, and fragmentation typically sets in before the CNTs reach lattice temperatures that would produce thermal damage. All figures show maxima and minima of SWNT stability with respect to laser intensity. Comparing Figs. 2 and 4, which show the response of the same SWNTs to laser pulses with identical parameters, but assuming different boundary conditions, we obtain that for fixed periodic boundary conditions the average damage threshold is lower. Especially the tubes with diameters below $d=8 \AA$ are more stable if they can expand longitudinally. For fixed boundary conditions, the $(16,0)$ tube with a diameter of $d=12.5 \AA$ shows the highest stability observed in the diameter range $d \in[4 ; 18 \AA]$. For variable boundary conditions, tubes of $d=5$ to $7 \AA$ diameter are as stable as the $(16,0)$ tube. Moreover, the $(14,0)$ SWNT with a diameter of $d=11.0 \AA$ even more stable. For both types of boundary conditions, the stability of the $(12,0)$ and $(13,0)$ CNTs with respect to laser excitation is relatively low (around $0.3 \mathrm{eV} /$ atom below the highest thresholds in the sample). If we now focus on the armchair SWNTs at $\tau$ $=50 \mathrm{fs}$ pulse duration [see Figs. 2(b) and 4(b)] we find a slightly simpler dependency of the damage thresholds on the tube diameter (although for a smaller sample of 7 tube diameters compared to the 17 diameters analyzed in the case of zigzag tubes). Specifically for fixed tube length [Fig. 2(b)], there is a clear stability maximum for the $(9,9)$ CNT of $d$
$=12.2 \AA$ diameter; both for lower and for higher diameters the stability falls monotonously. For variable periodic boundary conditions [Fig. 4(b)], the stability maximum is at $d$ $=13.6 \AA$ corresponding to the $(10,10)$ SWNT. Here the thresholds for the narrower tubes vary non-monotonically. Note, that the tubes with odd indices $(7,7)$ and $(9,9)$ show lower stability than the tubes with even indices $(6,6),(8,8)$, and $(10,10)$.

Another interesting difference between zigzag and armchair nanotubes is due to the different mechanical response along the tube axis for zigzag and armchair tubes [higher stiffness of the $(n, n)$ tubes in the direction perpendicular to its axis as compared to the $(n, 0)$ tubes]. In the case of zigzag tubes, close to but below the damage threshold, we observe vibrations of the nanotube walls that lead to deviations from the circular cross section creating a standing wave on the nanotube wall. This standing wave was not observed for armchair tubes. In Fig. 7 we have shown that for laser intensities slightly below threshold the spherical wall of the CNT deforms into a standing wave. In order to make this effect clearer, we show in Fig. 9 a plot of three consecutive CNT structures in which the atoms are represented as filled circles and projected onto the $x y$ plane. They correspond to a $(22,0)$ SWNT with $N=352$ atoms which has absorbed $E_{0}$ $=1.96 \mathrm{eV} /$ atom from a $\tau=20$ fs laser pulse. Each color corresponds to a section formed by a zigzag line of atoms along the zigzag CNT perimeter. The formation of a standing wave on the tube wall is clear. We quantify the deviations of the CNT wall from the perfect circle by fitting each of the eight 44 atom sections of the CNT to the nearest circle, i.e., by minimizing the function

$$
f\left(x_{0}, y_{0}, r\right)=\sum_{i=1}^{N_{\text {section }}}\left[\sqrt{\left(x_{i}-x_{0}\right)^{2}+\left(y_{i}-y_{0}\right)^{2}}-r\right]^{2}
$$

with respect to the circle center $\left(x_{0}, y_{0}\right)$ and radius $r$ for each section. The time evolution of the radii gained by this procedure is shown in Fig. 10. The radii of the eight tube sections oscillate in phase directly after laser excitation but after 2 ps some dephasing is seen. Now the oscillation amplitude of an atom $i$ with respect to the fitted circle can be defined as

$$
A(\boldsymbol{r})=\left|\sqrt{\left(x_{i}-x_{0}\right)^{2}+\left(y_{i}-y_{0}\right)^{2}}-r\right|
$$

and can be used to quantify the standing wave. The average of all of these amplitudes is plotted as a function of time in Fig. 11. This plot shows that the amplitude of the standing wave builds up with a time delay of approximately $400 \mathrm{fs}$ after the action of the pulse and comes to a maximum around $t=800 \mathrm{fs}$. Subsequently, the standing wave decays again on a time scale of several hundred femtoseconds (related to the coupling of the electrons to the optical phonons of the tube). We should note that electronic temperature reached in our simulations get closer to $T_{e}=20000 \mathrm{~K}$, while the ionic temperature which initially was $T_{l}=300 \mathrm{~K}$ increases due to electron-phonon coupling and reaches $T_{l}=500 \mathrm{~K}$. As can be seen from the time evolution of the SWNT radius during the formation of the standing wave (Fig. 10), the radial expansion immediately following the laser pulse is about $0.8 \AA$. This means that in a perfectly ordered SWNT bundle with 

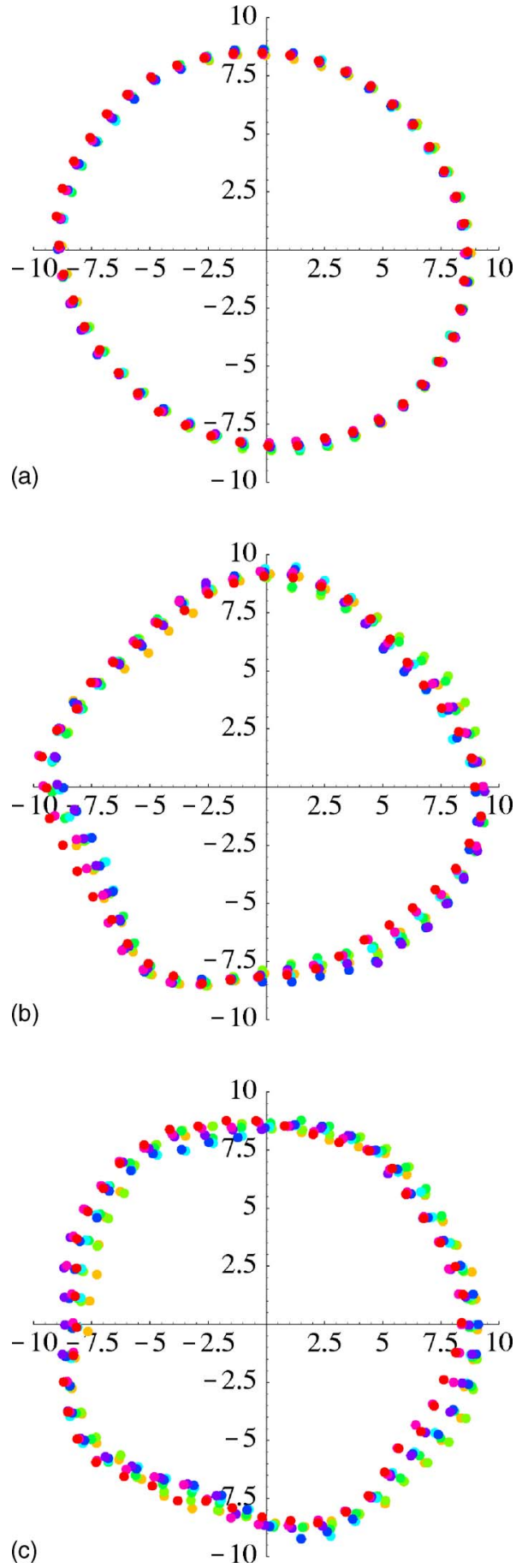

FIG. 9. (Color online) Selected structure of the $(22,0)$ nanotube with variable MD supercell excited just below the ablation threshold. Three examples of the standing wave, at $t=0,850$, and $1940 \mathrm{fs}$, respectively.

distances between nanotubes of $d=0.3 \mathrm{~nm},{ }^{41}$ an expansion of this magnitude would lead to a collision between neighboring tubes and thus a modified behavior not taken into account in our calculations. They should rather be taken to reflect the structural evolution of members of loose SWNT bundles where the calculated radial expansion does not yet lead to increased interactions between neigboring tubes.

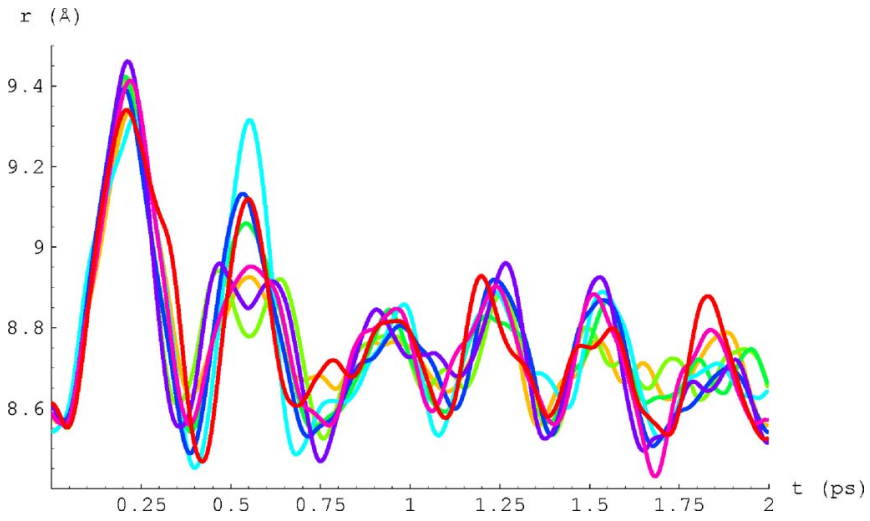

FIG. 10. (Color online) Analysis of a $(22,0)$ nanotube with variable MD supercell excited just below the ablation threshold. The radii of the sections oscillate in phase directly after laser excitation but after 2 ps some dephasing is seen. Each color corresponds to a section of the CNT as described in the text.

For the two principal chiralities we have considered, i.e. armchair and zigzag nanotubes, there are typical angles between CC bonds with respect to the CNT axis. In the case of an armchair CNT, we have one type of bond at a right angle to the tube axis (now called type I) and one type at an obtuse angle with the axis (now called type II). In the case of a zigzag CNT, there is a bond type parallel to the tube axis (type III), and one at an acute angle (type IV). For an illustration, see Fig. 12. In Fig. 13 we show an example of the time evolution of the average bond length for each of the armchair bond types. Its corresponds to a $(10,10)$ carbon nanotube excited by a laser pulse of $\tau=100$ fs duration during which it absorbs $E_{0}=2.17 \mathrm{eV} /$ atom. A first striking characteristic in Fig. 13(a) is the difference in amplitude of the average bond lengths which is signature of the radial nanotube expansion. Even though in this case of below-threshold excitation, both types of bonds relax to the same average bond length, it is clear that the type I bond would reach the breaking point before the type II bond if the laser intensity

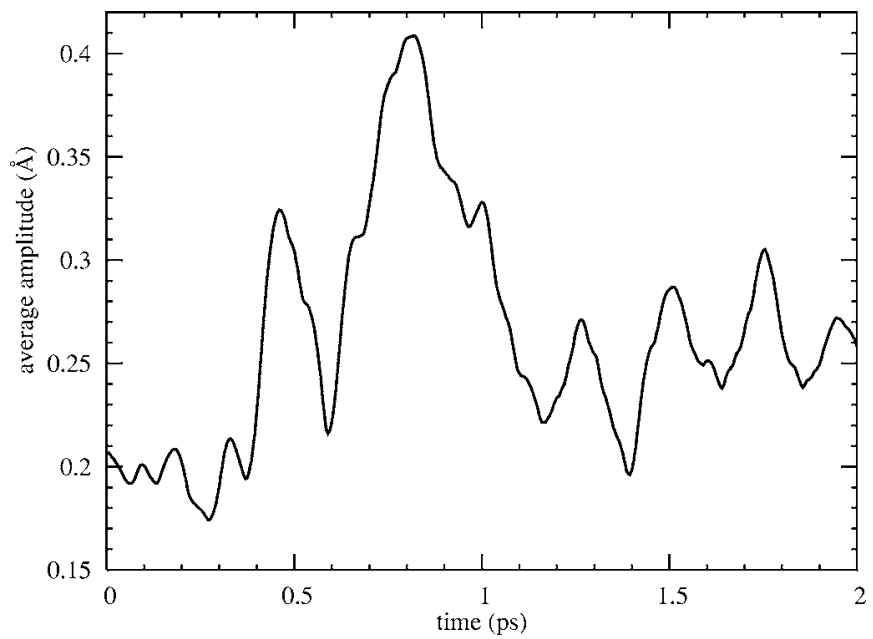

FIG. 11. Absolute value of the $(22,0)$ CNT deformation amplitude from Fig. 10, averaged over all sections of a CNT structure (for explanation see text), as a function of time. 


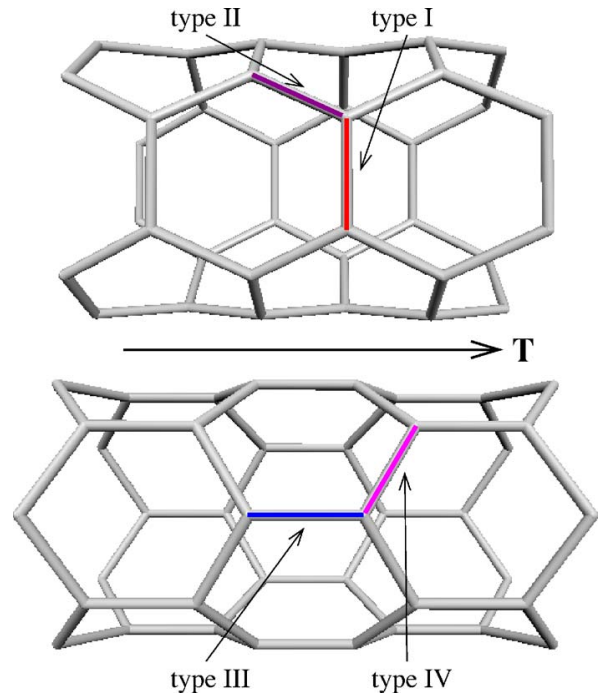

FIG. 12. (Color online) Orientation of the bonds in armchair (top) and zigzag (bottom) SWNTs with respect to the tube axis $\boldsymbol{T}$. The four type of bonds are stretched to a different extent in the intense breathing modes vibrations excited by a laser pulse.

were slightly increased. Consequently, we have observed that damage in armchair tubes starts with the breaking of type I bonds. After a first bond breaks, a cascade of bond breaking shows up as a tearing effect. Atoms at the edge of such a tear which now exhibit dangling bonds can leave the system rather easily. Thus, at laser intensities only slightly above the damage threshold, we often observe single carbon atoms leaving the carbon nanotube. In the case of zigzag CNTs, we find that both type III and type IV bond length averages oscillate in phase, at a high frequency similar to that of type II bonds in armchair CNTs [see Fig. 13(b)]. We observe that initially, type III bonds (along the tube axis) oscillate with larger amplitude than type IV bonds.

Finally, it is important to note that the lattice temperatures that are achieved at the damage threshold are on average $T_{l}$ $=750 \mathrm{~K}$. Thus, the fragmentation mechanisms we have presented here are predominantly due to the electron-hole excitation created by the laser pulse. At the damage threshold, we observe a peak excitation of approximately $\xi=8 \%$ of the electrons from the valence into the conduction band. This means that with the fragmentation conditions created by ultrashort laser pulses we are in the brittle regime and not in the superplastic regime involving lattice temperatures of $T_{l}$ $=2000 \mathrm{~K}$ reported in Ref. 43 .

\section{SUMMARY AND CONCLUSIONS}

In this work we have theoretically studied the structural response of SWNT of different diameters and chiralities to femtosecond laser excitation. In particular, the damage thresholds were carefully determined with the help of systematic MD simulations. Due to their metastable character carbon nanotubes (CNTs) may transform into more stable structures under the appropriate "annealing" conditions. Investigations of the stability and annealing pathways of metastable structures are of primary importance, specially in the
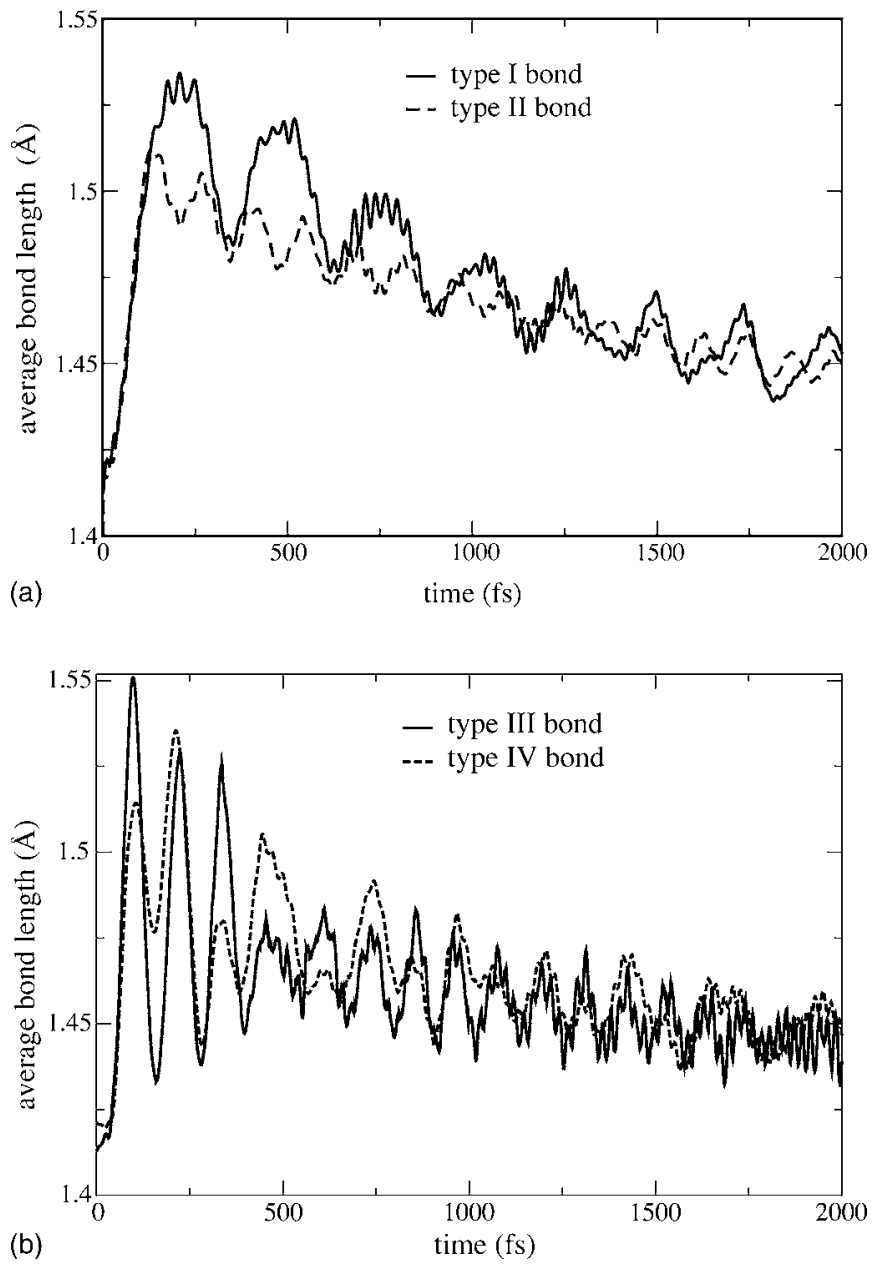

FIG. 13. (a) Average bond length for the two kinds of bonds in an armchair $(10,10)$ carbon nanotube, either perpendicular to the tube axis or not (see Fig. 12 for the definition of the bond types). The energy deposited by the $\tau=100 \mathrm{fs}$ laser pulse is $E_{0}=2.17 \mathrm{eV} /$ atom (well below the damage threshold, see Fig. 5). (b) Average bond length for the two kinds of bonds in a zigzag $(18,0)$ CNT, either along the tube axis or not. The energy absorbed from the $\tau=50 \mathrm{fs}$ laser pulse was $E_{0}=2.02 \mathrm{eV} /$ atom (just below the damage threshold).

context of technological applications. Coalescence of SWNTs is an example of structural transformation leading to a more stable structure, i.e., a nanotube of larger diameter. ${ }^{42}$ Thus we believe that this work is the first step for the understanding of those processes. Comparing two types of tube chiralities at the same laser pulse durations, we find that on averge SWNTs of zigzag and armchair type can withstand comparable laser intensities before breaking, but threshold dependencies on diameter differ.

The fact that carbon nanotubes have different damage thresholds as function of diameter is an important result of our simulations. It should be reflected in experiments performed on multiwall nanotubes, where the laser would be able to affect only some of the tubes. This might have important consequences for the manipulation of transport properties of multiwall nanotubes. Another important observation is the fact that nanotubes excited by laser intensities above the threshold tear open and can interact with similar or other 
tubes, which may lead to the creation of new types of structures. Finally, in the case of confined carbon nanostructures in carbon nanotubes, the laser could act differently on the confined nanostructures (for example fullerenes) than on the nanotube itself.

\section{ACKNOWLEDGMENTS}

H.O.J. gratefully acknowledges support from the DFG through the Emmy Noether Program. A.H.R. acknowledges the support of Conacyt Mexico under Grant No. J-42647-F. A.R. was supported by the NANOQUANTA Network of Excellence (Grant No. NMP4-CT-2004-500198) and SANES project (Grant No. NMP4-CT-2006-017310) and Information Society Technologies (IST) contract Number IST-200138051 (Project No. M-DNA). M.E.G. acknowledges support by the Deutsche Forschungsgemeinschaft (DFG) through the priority program No. SPP 1134 and by the European Community Research Training Network FLASH (Grant No. MRTN-CT-2003-503641).
*Electronic address: jeschke@itp.uni-frankfurt.de

${ }^{1}$ M. Terrones, Annu. Rev. Mater. Sci. 33, 413 (2003).

${ }^{2}$ R. Saito, G. Dresselhaus, and M. S. Dresselhaus, Physical Properties of Carbon Nanotubes (Imperial College Press, London, 1998).

${ }^{3}$ H. Rafii-Tabar, Phys. Rep. 390, 235 (2004).

${ }^{4}$ R. H. Baughman, A. A. Zakhidov, and W. A. de Heer, Science 297, 787 (2002), and references therein.

${ }^{5}$ P. G. Collins, K. Bradley, M. Ishigami, and A. Zettl, Science 287, 1801 (2000).

${ }^{6}$ A. Hashimoto, K. Suenaga, A. Gloter, K. Urita, and S. Iijima, Nature (London) 430, 870 (2004), and references therein.

${ }^{7}$ E. S. Choi, J. S. Brooks, D. L. Eaton, M. S. Al-Haik, M. Y. Hussaini, H. Garmestani, D. Li, and K. Dahmen, J. Appl. Phys. 94, 6034 (2003).

${ }^{8}$ Y.-C. Chen, H.-F. Cheng, Y.-S. Hsieh, and Y.-M. Ysau, J. Appl. Phys. 94, 7739 (2003).

${ }^{9}$ P. P. Vasil'ev, I. H. White, and J. Gowar, Rep. Prog. Phys. 63, 1997 (2000).

${ }^{10}$ U. Keller, Nature (London) 424, 831 (2003).

${ }^{11}$ H. M. Phillips and R. A. Sauerbrey, Opt. Eng. 32, 2424 (1993).

${ }^{12}$ A. H. Romero, M. E. Garcia, F. Valencia, H. Terrones, M. Terrones, and H. O. Jeschke, Nano Lett. 5, 1361 (2005).

${ }^{13}$ D. A. Akimov, M. V. Alfimov, S. O. Konorov, A. A. Ivanov, S. Botti, A. A. Podshivalov, R. Ciardi, L. De Dominicis, L. S. Asilyan, R. Fantoni, and A. M. Zheltikov, JETP 98, 220 (2004).

${ }^{14}$ Y.-Z. Ma, J. Stenger, J. Zimmermann, S. M. Bachilo, R. E. Smalley, R. B. Weisman, and G. R. Fleming, J. Chem. Phys. 120, 3368 (2004).

${ }^{15}$ F. Wang, G. Dukovic, L. E. Brus, and T. F. Heinz, Phys. Rev. Lett. 92, 177401 (2004).

${ }^{16}$ R. Z. Ma, B. Q. Wei, C. L. Xu, J. Liang, and D. H. Wu, Carbon 38, 623 (2000).

${ }^{17}$ P. Corio, P. S. Santos, M. A. Pimenta, and M. S. Dresselhaus, Chem. Phys. Lett. 360, 557 (2002).

${ }^{18}$ M. S. Dresselhaus, G. Dresselhaus, R. Saito, and A. Jorio, Phys. Rep. 409, 47 (2005).

${ }^{19}$ M. Yudasaka, T. Ichihashi, D. Kasuya, H. Kataura, and S. Iijima, Carbon 41, 1273 (2003).

${ }^{20}$ Z. M. Li, Z. K. Tang, H. J. Liu, N. Wang, C. T. Chan, R. Saito, S. Okada, G. D. Li, J. S. Chen, N. Nagasawa, and S. Tsuda, Phys. Rev. Lett. 87, 127401 (2001); N. Wang, Z. K. Tang, G. D. Li, and J. S. Che, Nature (London) 408, 50 (2000); A. G. Marinopoulos, L. Reining, A. Rubio, and N. Vast, Phys. Rev. Lett. 91, 046402 (2003); Appl. Phys. A 78, 1157 (2004); C. L.
Kane and E. J. Mele, Phys. Rev. Lett. 90, 207401 (2003); C. D. Spataru, S. Ismail-Beigi, L. X. Benedict, and S. G. Louie, ibid. 92, 077402 (2004); E. Chang, G. Bussi, A. Ruini, and E. Molinari, ibid. 92, 196401 (2004).

${ }^{21}$ Y. Miyamoto, N. Jinbo, H. Nakamura, A. Rubio, and D. Tománek, Phys. Rev. B 70, 233408 (2004).

${ }^{22}$ Y. Miyamoto, A. Rubio, S. Berber, M. Yoon, and D. Tománek, Phys. Rev. B 69, 121413(R) (2004); Y. Miyamoto, S. Berber, M. Yoon, A. Rubio, and D. Tománek, Chem. Phys. Lett. 392, 209 (2004).

${ }^{23}$ T. Dumitrica, M. E. Garcia, H. O. Jeschke, and B. I. Yakobson, Phys. Rev. Lett. 92, 117401 (2004).

${ }^{24}$ H. O. Jeschke and M. E. Garcia, in Nonlinear Optics, Quantum Optics and Ultrafast Phenomena with X-rays, edited by Bernhard W. Adams (Kluwer Academic Publishers, Boston, 2003).

${ }^{25}$ H. O. Jeschke, M. E. Garcia, and K. H. Bennemann, Phys. Rev. Lett. 87, 015003 (2001).

${ }^{26}$ H. O. Jeschke and M. E. Garcia, Appl. Surf. Sci. 197-198, 107 (2002); M. E. Garcia and H. O. Jeschke, ibid. 208-209, 61 (2003).

${ }^{27}$ A. H. Romero, M. E. Garcia, and H. O. Jeschke, Appl. Phys. A 79, 899 (2004).

${ }^{28}$ J. C. Tully, J. Chem. Phys. 93, 1061 (1990).

${ }^{29}$ C. F. Craig, W. R. Duncan, and O. V. Prezhdo, Phys. Rev. Lett. 95, 163001 (2005).

${ }^{30}$ P. V. Parandekar and J. C. Tully, J. Chem. Phys. 122, 094102 (2005).

${ }^{31}$ O. V. Prezhdo and P. J. Rossky, J. Chem. Phys. 107, 825 (1997).

${ }^{32}$ O. V. Prezhdo, J. Chem. Phys. 111, 8366 (1999).

${ }^{33}$ A. W. Jasper, S. Nangia, C. Zhu, and D. G. Truhlar, Acc. Chem. Res. 39, 101 (2006).

${ }^{34}$ H. C. Andersen, J. Chem. Phys. 72, 2384 (1980).

${ }^{35}$ M. Parrinello and A. Rahman, Phys. Rev. Lett. 45, 1196 (1980).

${ }^{36}$ C. H. Xu, C. Z. Wang, C. T. Chan, and K. M. Ho, J. Phys.: Condens. Matter 4, 6047 (1992); I. Kwon, R. Biswas, C. Z. Wang, K. M. Ho, and C. M. Soukoulis, Phys. Rev. B 49, 7242 (1994).

${ }^{37}$ H. O. Jeschke, M. E. Garcia, and K. H. Bennemann, J. Appl. Phys. 91, 18 (2002).

${ }^{38}$ K. Urita, K. Suenaga, T. Sugai, H. Shinohara, and S. Iijima, Phys. Rev. Lett. 94, 155502 (2005).

${ }^{39}$ M. J. López, A. Rubio, J. A. Alonso, S. Lefrant, K. Méténier, and S. Bonnamy, Phys. Rev. Lett. 89, 255501 (2002).

${ }^{40}$ F. Valencia, A. H. Romero, H. O. Jeschke, and M. E. Garcia, Phys. Rev. B 74, 075409 (2006). 
${ }^{41}$ K. Miura, M. Ishikawa, R. Kitanishi, M. Yoshimura, K. Ueda, Y. Tatsumi, and N. Minami, Appl. Phys. Lett. 78, 832 (2001).

${ }^{42}$ P. Nikolaev, A. Thess, A. G. Rinzler, D. T. Colbert, and R. E. Smalley, Chem. Phys. Lett. 266, 422 (1997).
${ }^{43}$ J. Y. Huang, S. Chen, Z. Q. Wang, K. Kempa, Y. M. Wang, S. H. Jo, G. Chen, M. S. Dresselhaus, and Z. F. Ren, Nature (London) 439, 281 (2006). 